\title{
A Revelação de Abuso Sexual: As Medidas Adotadas pela Rede de Apoio ${ }^{1}$
}

\author{
Luísa Fernanda Habigzang ${ }^{2}$ \\ Michele da Silva Ramos \\ Sílvia Helena Koller \\ Universidade Federal do Rio Grande do Sul
}

\begin{abstract}
RESUMO - O presente estudo identificou e analisou as medidas adotadas pela rede de apoio de crianças e adolescentes após a revelação de abuso sexual. Participaram 40 meninas, entre oito e 16 anos, vítimas de abuso sexual. As medidas de proteção adotadas pela rede foram mapeadas através de entrevista semi-estruturada. A revelação foi feita aos pais em $42,5 \%$ da amostra e $92,5 \%$ das pessoas acreditaram. O abrigamento ocorreu em 35\% dos casos e o restante permaneceu com a família que afastou o agressor. A atitude de confiança da família na revelação e a denúncia da violência constituíram-se em um fator de proteção. Contudo, o alto índice de abrigamento e o não acompanhamento efetivo do afastamento do agressor representaram fatores de risco.
\end{abstract}

Palavras-chave: abuso sexual; rede de apoio; crianças; adolescentes.

\section{The Disclosure of Sexual Abuse: Adopted Measures by the Support Network}

\begin{abstract}
This study aimed to identify the measures adopted by the social and emotional support network of children and adolescents after disclosure of sexual abuse. Forty victims of sexual abuse, eight to sixteen years old girls, responded to an interview that mapped the measures adopted by the network. Disclosure to the parents took place in $42.5 \%$ of the cases, and $92.5 \%$ of the parents believed it to be true. $35 \%$ of the girls went to a shelter home and the rest remained with the family after exclusion of the aggressor. The trustworthiness of the family in disclosure and reporting of violence was a protective factor. However, the high shelter rate and absence of effective monitoring of the aggressor removal were risk factors.
\end{abstract}

Keywords: sexual abuse; network support; children; adolescents

O abuso sexual é um problema de saúde pública, definido como todas as formas de atividades sexuais, nas quais, as crianças e adolescentes não têm condições maturacionais e psicobiológicas de enfrentamento, transgredindo assim, as normas sociais, morais e legais. $\mathrm{O}$ abuso pode se estabelecer em relação homossexual ou heterossexual, no qual o agressor está em estágio psicossexual mais avançado que a vítima (Ministério da Saúde, 2002). O abuso pode ocorrer contra a vontade da criança ou adolescente ou pela indução de sua vontade, através das relações de poder e confiança entre a vítima e o agressor, bem como, pelo uso de violência física ou psicológica (ameaças e barganhas). O objetivo dos abusos sexuais é a gratificação sexual do agressor (Gomes, Junqueira, Silva, \& Junger, 2002).

O abuso sexual pode ocorrer em dois diferentes contextos, o intrafamiliar e o extrafamiliar. $\mathrm{O}$ abuso intrafamiliar ou incestuoso ocorre dentro do ambiente doméstico, no qual o abusador exerce uma função de confiança, cuidado e poder em relação à criança. $\mathrm{O}$ abuso extrafamiliar é perpetrado fora das relações familiares, envolvendo, por exemplo, vizinhos ou desconhecidos e os casos de pornografia infantil e exploração sexual comercial (Habigzang \& Caminha, 2004; Koller \& De Antoni, 2004).

O tempo de duração do abuso sexual está relacionado com o contexto em que ocorre a violência. Abusos sexuais extrafamiliares tendem a ter menor tempo de duração em

1 Apoio CNPq.

2 Endereço para correspondência: Instituto de Psicologia - UFRGS, Rua Ramiro Barcelos, $\mathrm{n}^{\circ}$ 2600, sala 104, Porto Alegre, RS. CEP: 90035-003. Fone: (51) 33165150.E-mail: habigzang.luisa@gmail.com comparação aos abusos ocorridos no contexto intrafamiliar, pois a criança necessita de um tempo maior para perceber que os comportamentos do agressor são abusivos (Sharma \& Gupta, 2004). Fatores como a dinâmica do segredo e o vínculo próximo com o agressor também contribuem para maior duração dos abusos intrafamiliares. Em muitos casos, as vítimas conseguem revelar a violência sofrida apenas na adolescência ou na vida adulta, devido ao medo de serem culpabilizadas pelo abuso e responsabilizadas pela desestruturação da família, assim como pelo medo de que o agressor cumpra as ameaças realizadas para manter a violência em segredo (Berliner \& Conte, 1995).

A análise de aspectos relacionados à revelação de abuso sexual foi realizada a partir dos arquivos referentes a 218 casos de crianças e adolescentes, em órgãos de proteção responsáveis pelo atendimento destes (Goodman-Brown, Edelstein, Goodman, Jones, \& Gordon, 2003). A idade da vítima no início do abuso apareceu correlacionada com o tempo que esta levou para revelar o abuso, sendo que as crianças mais novas levaram um período maior para revelar a violência. $O$ gênero da vítima não apresentou relação com o tempo para a revelação da situação abusiva. No entanto, as crianças vítimas de violência intrafamiliar levaram um período maior para contar sobre o abuso quando comparadas com as crianças vítimas de violência extrafamiliar. As crianças que acreditavam nas possíveis conseqüências negativas quando contassem sobre o abuso, demonstraram um período mais longo para revelar o abuso sexual. O medo dessas crianças em relação às conseqüências negativas concentrou-se, principalmente, em danos à pessoas significativas, como 
por exemplo, familiares, estando também relacionada com o medo de conseqüências negativas a si própria ou aos agressores. A idade, o tipo de abuso (intrafamiliar ou extrafamiliar), o medo de conseqüências negativas, e a percepção de responsabilidade frente ao abuso estão relacionados com o período que as vítimas levam para revelar a violência sexual (Goodman-Brown et al., 2003).

A revelação do abuso sexual é um momento crucial para a vítima, pois pode gerar revitimizações caso os adultos não acreditem em seu relato e tomem as medidas protetivas cabíveis. A rede de apoio social e afetiva da criança pode minimizar ou potencializar os danos do abuso sexual no momento em que a criança consegue romper o segredo e revelar a violência. Tal rede é compreendida como o conjunto de sistemas e de pessoas significativas (estrutura) que compõem os relacionamentos existentes e percebidos pela criança, e que podem atuar no sentido de efetivamente protegê-la (função). Portanto, o apoio social e afetivo está relacionado à percepção que a pessoa possui de seu mundo social, como se orienta nele, suas estratégias e competências para formar vínculos, e com os recursos que esse lhe oferece frente a situações de risco que se apresentam ao longo do desenvolvimento (Brito \& Koller, 1999). Nos casos de abuso sexual contra crianças e adolescentes compreende-se como constituinte da rede: família, escola, comunidade, Conselho Tutelar, Delegacia, Conselho de Direitos da Criança, Ministério Público e Juizado da Infância e Adolescência, abrigos, serviços de saúde (postos de saúde e hospitais) e assistência social (Centro de Referência da Assistência Social e Centro de Referência Especializado da Assistência Social).

Os profissionais e as instituições que constituem a rede de apoio social para crianças e famílias vítimas de abuso sexual encontram-se diante do desafio de planejar intervenções efetivas que protejam a criança e minimizem os efeitos da violência sofrida (Ferreira \& Schramm, 2000). Entretanto, o despreparo da rede é ainda constatado, envolvendo desde os profissionais da área de saúde, educadores, assistentes sociais, juristas até as instituições escolares, hospitalares e jurídicas, em manejar e tratar adequadamente os casos surgidos. O trabalho nesse campo é fragmentado e desorganizado (Amazarray \& Koller, 1998).

A organização e a eficácia das redes de apoio às crianças e aos adolescentes vítimas de abuso sexual foram avaliadas através de uma pesquisa documental, na qual foram analisados todos os expedientes de casos de violência sexual ajuizados pela Coordenadoria das Promotorias da Infância e Juventude de Porto Alegre no período de 1992 a 1998 (Habigzang, Azevedo, Koller \& Machado, 2005, 2006). Os resultados apontaram que $46,8 \%$ das vítimas foram abusadas entre os cinco e 10 anos, $80,9 \%$ eram do sexo feminino e $42,6 \%$ das vítimas revelaram o abuso na adolescência. A pessoa que mais denunciou o abuso aos órgãos de proteção foi a mãe da vítima (37,6\% dos casos). Em 94,8\% dos casos os abusadores eram homens que conviviam na residência da criança e possuíam uma relação de confiança e cuidado com ela. Também foram identificadas outras formas de violência, tais como negligência, abusos físicos e psicológicos. Foi constatado que, na maioria dos casos, a violência sexual já era do conhecimento dos familiares, entretanto a denúncia se efetivou por motivos diversos do ato em si.
Em relação ao atendimento efetuado pela rede, ficou evidente que o abuso sexual foi ignorado, sendo que as intervenções se deram em função de outras violações. Além disso, foi verificada a falta de comunicação entre os órgãos que compuseram a rede. Desta forma, não houve acompanhamento, avaliação e atendimento adequados. Além disso, os agressores, com poucas exceções, foram punidos criminalmente. Na maioria dos casos analisados, as crianças foram abrigadas e o(s) pai(s) destituído(s) do poder familiar (ex-pátrio poder). O estudo apontou a necessidade emergente de criar serviços especializados de atendimento e capacitar os profissionais que trabalham com essas crianças e com suas famílias, permitindo-lhes obter uma compreensão real dos casos, bem como conduzir uma intervenção adequada (Habigzang, Azevedo, Koller, \& Machado, 2005, 2006).

Outro estudo realizado a partir dos prontuários registrados em dois conselhos tutelares da cidade de Feira de Santana no estado da Bahia, no período entre janeiro de 2003 e dezembro de 2004 estimou a prevalência de violência contra crianças e adolescentes (Costa et al., 2007). Foram avaliados 1.293 casos de violência, com uma média de 24 denúncias por mês, para o Conselho I, e de 29 casos por mês, para o Conselho II, totalizando 297 e 349 ocorrências por ano. Dentre os casos de violência contra crianças, $78 \%$ ocorreram nos domicílios destas. A notificação foi realizada anonimamente em 30,8\%, pela mãe em $21,2 \%$, pelo pai em $14,92 \%$ e por outros em $8,5 \%$. Os casos de violência sexual foram 68 , sendo 10 deles envolvendo exploração sexual e $80 \%$ das vítimas eram do sexo feminino.

O principal agressor da violência sexual tinha vínculos familiares com as vítimas. Foi verificado que em 29\% dos casos de abuso sexual não havia registro nos prontuários que identificasse o agressor. Os encaminhamentos à rede de atendimento foram realizados de acordo com a violência notificada. Foram encaminhados 487 casos, entre os quais 216 não constavam informações referentes a esses procedimentos, o que impossibilitou a análise. Esses resultados sugeriram que os conselhos tutelares estavam realizando um trabalho fragmentado e sem acompanhamento efetivo dos casos que foram encaminhados para a rede de atendimento. Esse estudo apontou a importância de incluir um Sistema de Referência e Contra-Referência das instituições de atendimento da rede do município para o acompanhamento dos casos pelos conselhos tutelares (Costa et al., 2007).

Em uma revisão da literatura realizada por Maia e Williams (2005) sobre os fatores de risco e de proteção para o desenvolvimento infantil, considerando a rede de atendimento em situações de violência, foi verificada a importância da capacitação dos profissionais das áreas social, saúde e educação para a identificação e encaminhamentos adequados frente à violência contra crianças e adolescentes. A capacitação dos conselheiros tutelares também foi apontada como fundamental em relação aos fatores de risco e proteção e indicadores de abuso sexual, pois tais profissionais são os principais responsáveis por receber as notificações dos casos nos quais existe suspeita ou confirmação de violência contra criança ou adolescentes.

A avaliação efetiva dos indicadores de violência intrafamiliar é de extrema importância. Os profissionais devem estar capacitados quanto à realidade histórica da vítima para 
identificar a rede de apoio social e afetiva que a criança dispõe para protegê-la e afastá-la de situações de risco. Os profissionais que atuam com crianças e adolescentes em situações de risco devem considerar todo o seu contexto (desde a família nuclear até a sociedade ampla) ambiental e situacional, pois estes podem ter influência no desenvolvimento das vítimas (Koller \& De Antoni, 2004).

Dentre as medidas para a proteção da criança que devem ser adotadas pela rede está a notificação do abuso sexual aos órgãos de proteção (Conselho Tutelar, Delegacias e Ministério Público), pois contribui para combater a violência no caso individual da criança vítima e auxilia a compreender tal fenômeno, considerando os índices epidemiológicos que se tornam mais fidedignos (Amazarray \& Koller, 1998). O Estatuto da Criança e do Adolescente (ECA, Estatuto da Criança e do Adolescente, Lei Federal n ${ }^{\circ}$ 8.069, Brasil, 1990) determina como obrigatória a notificação de casos suspeitos ou confirmados de maus-tratos contra a criança ou adolescente e o abuso sexual é uma das formas mais graves de maus-tratos. A principal preocupação em uma intervenção deve ser a avaliação da capacidade da família de proteger a criança de novos abusos e a necessidade ou não do afastamento imediato da criança (Zavaschi, Telelbom, Gazal \& Shansis 1991). A proteção da vítima pode ocorrer através de dois caminhos: o afastamento do abusador ou o afastamento da vítima.

A compreensão das medidas adotadas pela rede de apoio social e afetiva diante a revelação de abuso sexual é fundamental para a identificação de práticas que potencializam o risco e o dano da violência para a vítima, assim como para o planejamento de estratégias mais eficazes para a promoção da proteção e qualidade de vida das crianças e adolescentes. A rede de apoio estará sendo efetiva e contribuindo para minimizar os efeitos do abuso sexual quando oferecer credibilidade ao relato de abuso da criança, protegê-la do agressor, evitando novos episódios de violência, der suporte afetivo para a vítima, denunciar a situação aos órgãos de proteção e garantir o acompanhamento médico, psicológico, social e jurídico necessários à criança e sua família.

O presente artigo teve como objetivo identificar e analisar os fatores de risco e de proteção nas medidas adotadas pela rede de apoio social e afetiva em situações de revelação de abuso sexual contra crianças e adolescentes. A compreensão de rede adotada neste estudo incluiu a família e demais instituições que compuseram a rede de atendimento. A análise de tais medidas foi realizada a partir de casos clínicos de meninas vítimas de abuso sexual intrafamiliar e extrafamiliar de uma cidade da região metropolitana de Porto Alegre/ RS. As medidas adotadas foram investigadas através de entrevistas com a própria criança ou adolescente e com um cuidador não-abusivo.

\section{Método}

\section{Participantes}

Participaram deste estudo 40 meninas com idade entre nove e 16 anos $(m=11,28, S D=1,89)$, residentes em uma cidade da região metropolitana de Porto Alegre/ RS. O critério de inclusão foi a presença de pelo menos um episódio de abuso sexual. $\mathrm{O}$ abuso sexual ocorreu no contexto familiar em 30 casos (75\%), fora do contexto familiar em seis casos $(15 \%)$ e em quatro casos $(10 \%)$ as meninas foram vitimizadas por familiares e vizinhos. A forma de abuso sexual envolveu contato físico sem estupro em $65 \%$ dos casos, estupro em $30 \%$ e assédio verbal ou exibicionismo, sem contato físico em $5 \%$. A idade de início do abuso sexual ocorreu em média aos 8,97 anos $(S D=2,12)$. A duração da violência foi de mais de um ano em 37,5\% dos casos. Em 82,5\% dos casos houve episódios múltiplos de abuso e em $17,5 \%$ a violência se configurou como episódio único.

\section{Instrumento e procedimentos}

O projeto de pesquisa foi submetido e aprovado pelo Comitê de Ética da Universidade Federal do Rio Grande do Sul. As participantes foram encaminhadas pelo Conselho Tutelar, abrigos, Juizado da Infância e Juventude e Programa Sentinela do município no qual a pesquisa foi realizada. As participantes foram incluídas mediante a assinatura do Termo de Consentimento Livre e Esclarecido de seus responsáveis e pelo seu consentimento.

O instrumento utilizado para levantamento de dados foi uma entrevista semi-estruturada constituída por duas partes: na primeira parte, o objetivo foi estabelecer um vínculo com a participante, criando um espaço seguro baseado em uma relação de confiança. A segunda parte foi baseada na entrevista publicada pelo The Metropolitan Toronto Special Committee on Child Abuse (1995), traduzida para o português e adaptada por Kristensen (1996). Esta entrevista teve como objetivos coletar dados biosociodemográficos, obter o relato da participante com relação ao abuso sexual, mapear a freqüência e a dinâmica dos episódios abusivos, identificar fatores de risco e proteção na rede de apoio social e afetiva. A entrevista foi realizada em um ou dois encontros com as meninas. Após o processo de avaliação todas as participantes foram encaminhadas para acompanhamento psicológico. Para este estudo a análise da entrevista centrou-se nas questões que investigaram a ação da rede, tais como: se a vítima contou para alguém o que estava acontecendo; para quem contou; se ela sofreu algum tipo de ameaça para não contar sobre o abuso; o que aconteceu depois que revelou a violência sexual; como sua família reagiu e o que fez depois que contou sobre o abuso; se ela foi à delegacia ou ao Conselho Tutelar falar sobre o que ocorreu; o que aconteceu depois de ir a estes lugares; como está a sua vida agora (Habigzang et al., 2008).

\section{Resultados e Discussão}

A entrevista utilizada possibilitou o mapeamento de informações sobre as participantes e a história do abuso sexual. Para este estudo, foram analisadas as informações acerca dos fatores de risco na família, sobre a revelação do abuso e medidas adotadas pela rede de apoio social e afetiva. 


\section{Fatores de Risco na Família}

Considerando que a rede de apoio é também constituída pela família, foram analisados fatores de risco neste contexto que contribuíram para o abuso sexual e situação da revelação da violência. Verificou-se que, 40\% dos agressores utilizavam álcool ou drogas e em $40 \%$ dos casos havia situação de desemprego familiar. A presença de padrastos ou madrastas foi constatada em $55 \%$ dos casos. O abusador era responsável pelos cuidados da vítima em $47,5 \%$ dos casos e $42,5 \%$ dos agressores já havia vitimizado sexualmente ou fisicamente outras pessoas. Estes fatores estão freqüentemente associados com a violência sexual, principalmente no contexto familiar (Furniss, 1993; Habigzang et al., 2005; Sanderson, 2005). A Tabela 1 apresenta informações sobre a família das participantes.

A entrevista buscou mapear quem foram os abusadores e em alguns casos foi identificada a presença de mais de um agressor. Os resultados apontam que os pais $(25 \%)$, padrastos $(22,5 \%)$ e tios $(17,5 \%)$ foram os principais agressores. Em nenhum caso, mesmo naqueles que se configuraram como extrafamiliares, o agressor era completamente desconhecido para a vítima. Estes dados estão em consonância com outros estudos (Braun, 2002; Costa et al., 2007; Habigzang et al., 2005; Sanderson, 2005) e demonstram que o abuso sexual é um risco perpetrado por pessoas conhecidas das vítimas e de suas famílias que utilizam-se da relação de confiança e amizade para cometerem essa violência. Em 95,9\% dos casos, os agressores eram do sexo masculino e apenas $4,1 \%$ eram do sexo feminino. As mulheres identificadas como agressoras participaram da violência auxiliando seus com-

Tabela 1. Freqüências e Percentagens sobre Fatores de Risco Presentes nas Famílias.

\begin{tabular}{lcc}
\hline Informações sobre a família & $f$ & $\%$ \\
\hline Agressor usava álcool elou drogas & & \\
\hline Sim & 16 & 40 \\
\hline Não & 24 & 60 \\
\hline Presença de desemprego na família & 16 & 40 \\
\hline Sim & 24 & 60 \\
\hline Não & & \\
\hline Família reestruturada & 22 & 55 \\
\hline Sim & 18 & 45 \\
\hline Não & & \\
\hline Abuso sexual da mãe na infância & 04 & 10 \\
\hline Sim & 08 & 20 \\
\hline Não & 28 & 70 \\
\hline Sem informação & & \\
\hline Abusador cuidava da criança & 19 & 47,5 \\
\hline Sim & 21 & 52,5 \\
\hline Não & & \\
\hline Abusador agrediu sexualmente outras vítimas & & \\
\hline Sim & & \\
\hline Não & & \\
\hline
\end{tabular}

panheiros a cometerem o abuso. A Tabela 2 apresenta quem foram os agressores.

\section{Revelação do Abuso e Medidas Adotadas pela Rede de Atendimento}

Os resultados apontaram que as meninas revelaram o abuso aos pais em $42,5 \%$ e a outro familiar em $12,5 \%$. Estes dados estão de acordo com os estudos de Costa et al. (2007) e Habigzang et al. $(2005,2006)$ que verificaram que a denúncia foi comumente realizada por um familiar a quem a criança revelou a situação. A maioria das pessoas (92,5\%) acreditou na revelação da situação de abuso, porém $10 \%$ destas não adotou nenhuma atitude protetiva. As medidas de proteção adotadas pelas famílias foram a denúncia e o afastamento dos agressores. Contudo, em 32,5\% dos casos os familiares não realizaram uma denúncia formal da violência aos órgãos de proteção, mantendo uma situação de vulnerabilidade para a criança. $\mathrm{O}$ fato de não realizar uma denúncia significa um fator de risco para a revitimização da criança ou adolescente, bem como contribui para a impunidade dos agressores (Sanderson, 2005). Além disso, esta atitude da família contribui para a crença da criança de que ela é a responsável pela violência e não os agressores. Apesar de acreditar na revelação da criança, muitas famílias não tiveram uma postura protetiva. Isto se deve a diferentes situações: medo de outras formas de violência pelos agressores, falta de conhecimento das leis de proteção a criança e banalização da violência. Para algumas famílias participantes deste estudo o abuso sexual ocorreu apenas quando identificado estupro e muitas apresentaram a crença de que a denúncia não resolve a situação, pois os agressores dificilmente são penalizados e a família permanece em risco.

$\mathrm{O}$ abrigamento das meninas ocorreu em $35 \%$ dos casos e o restante permaneceu convivendo com a família. A permanência das meninas com as famílias ocorreu nos casos em que o agressor foi afastado do convívio com a criança e tal medida foi cumprida pelos demais familiares. Apenas $20 \%$ dos agressores foram punidos criminalmente. A impu-

Tabela 2. Freqüências e Percentagens sobre os Agressores

\begin{tabular}{lccc}
\hline Agressor & $f^{\mathrm{a}}$ & $\%$ & $\%$ casos \\
\hline Pai & 10 & 20,4 & 25,0 \\
\hline Padrasto & 9 & 18,4 & 22,5 \\
\hline Tio & 7 & 14,3 & 17,5 \\
\hline Avô & 4 & 8,2 & 10,0 \\
\hline Irmão & 4 & 8,2 & 10,0 \\
\hline Vizinho & 4 & 8,2 & 10,0 \\
\hline Amigo & 5 & 10,2 & 12,5 \\
\hline Vodrasto & 1 & 2,0 & 2,5 \\
\hline Cunhado & 2 & 4,1 & 5,0 \\
\hline Padrinho & 1 & 2,0 & 2,5 \\
\hline Mãe & 1 & 2,0 & 2,5 \\
\hline Madrinha & 1 & 2,0 & 2,5 \\
\hline Total & 49 & $100,0 \%$ & $122,5 \%$ \\
\hline
\end{tabular}


nidade criminal do agressor pode estar associada à exigência de provas materiais do crime, ainda solicitada por alguns juízes, possíveis apenas nos casos em que ocorre estupro (Sanderson, 2005).

Estes dados apontam que as crianças e adolescentes receberam credibilidade dos adultos e que alguma medida protetiva, ou seja, afastamento do agressor e/ou notificação, foi adotada pela rede na maioria dos casos. O abrigamento das vítimas é ainda uma medida frequentemente adotada e a impunidade dos agressores um fato comum. Dessa forma, as crianças são retiradas de suas casas, enquanto que os perpetradores da violência permanecem impunes (Habigzang et al., 2005; 2006). Esta medida pode ser interpretada pelas crianças e suas famílias como uma punição para a própria vítima. O Estatuto da Criança e do Adolescente (ECA, Brasil, 1990) prevê o afastamento dos agressores da moradia comum. Contudo, não há um acompanhamento efetivo desta medida e o abrigamento para alguns casos é a única garantia de segurança para a criança.

A retirada da criança da família é, sem dúvida, uma intervenção radical; uma interdição que, como tal, pode se caracterizar como medida de tratamento da família e como medida protetiva que pode ser considerada para determinadas crianças ou adolescentes já como terapêutica, ou seja, reparadora. A criança ou o adolescente encaminhado para um abrigo necessita de acompanhamento profissional para não compreender essa decisão como uma forma de punição, que reforça a crença de que é responsável pelo abuso. $\mathrm{O}$ abrigo pode ser um lugar privilegiado para casos extremos, garantindo cuidados essenciais e rotinas definidas que viabilizem o desenvolvimento saudável de crianças e de adolescentes. Contudo, o abrigamento deve ser uma medida provisória e a criança tem o direito de conviver em família (Fromer, 2002). A Tabela 3 apresenta informações sobre as medidas adotadas pela rede frente à revelação do abuso sexual.

Outra medida de proteção significativa da rede foi o encaminhamento para atendimento psicológico. A maioria dos casos analisados foi encaminhada pelo Conselho Tutelar ou Ministério Público como medida de proteção através de ofícios. A avaliação e acompanhamento psicológico são fundamentais, uma vez que crianças e adolescentes vítimas de abuso sexual podem apresentar alterações cognitivas, afetivas e comportamentais significativas. Além disso, a literatura tem apontado a presença de transtornos de humor, de ansiedade, e disruptivos em crianças e adolescentes vítimas dessa forma de violência (Briere \& Elliott, 2003; Cohen,2001; Duarte \& Arboleda, 2004; Habigzang \& Caminha, 2004; Maniglio, 2009; Muthi \& Espelage, 2005; Runyon \& Kenny, 2002).

O tempo de espera por atendimento psicológico também foi avaliado, uma vez que a maioria das participantes estava em filas de espera aguardando atendimento em serviços da rede pública quando foram convidadas a participar do estudo. Apenas 25\% das meninas receberam atendimento psicológico até um mês após a revelação do abuso. Em 55\% dos casos as meninas estavam aguardando de um a seis meses por atendimento e $20 \%$ aguardavam por mais de seis meses. $\mathrm{O}$ atendimento psicológico imediato após a revelação do abuso sexual pode contribuir para a minimização do impacto desta experiência para o desenvolvimento de crianças e adolescentes (Elliott \& Carne, 2001; Runyon \& Kenny, 2002;
Tabela 3. Freqüência e Percentagens Relativas à Funcionalidade e Fatores da Rede da Apoio Social e Afetiva na Revelação do Abuso Sexual

\begin{tabular}{|c|c|c|}
\hline Sobre a revelação & $f$ & $\%$ \\
\hline \multicolumn{3}{|l|}{ Pessoa a quem revelou } \\
\hline Pais & 17 & 42,5 \\
\hline Outro familiar & 5 & 12,5 \\
\hline Amigo & 4 & 10 \\
\hline Escola & 04 & 10 \\
\hline Outro profissional & 04 & 10 \\
\hline Delegacia ou Conselho Tutelar & 02 & 5 \\
\hline Denúncia anônima de outros & 04 & 10 \\
\hline \multicolumn{3}{|l|}{ A pessoa acreditou } \\
\hline Sim & 37 & 92,5 \\
\hline Não & 03 & 7,5 \\
\hline \multicolumn{3}{|l|}{ Reação após a revelação } \\
\hline Denúncia & 05 & 12,5 \\
\hline Afastamento do agressor & 09 & 22,5 \\
\hline Denúncia e afastamento do agressor & 22 & 55 \\
\hline Atitude não protetiva & 04 & 10 \\
\hline \multicolumn{3}{|l|}{ Abrigamento } \\
\hline Sim & 14 & 35 \\
\hline Não & 24 & 60 \\
\hline Passou a residir com outro familiar & 02 & 5 \\
\hline \multicolumn{3}{|l|}{ Penalização do agressor } \\
\hline Sim & 08 & 20 \\
\hline Não & 32 & 80 \\
\hline
\end{tabular}

Saywitz, Mannarino, Berliner, \& Cohen, 2000). Além disso, contribui para o acompanhamento de medidas de proteção determinadas pelo Conselho Tutelar e Juizado da Infância e Adolescência. Dessa forma, a reorganização da rede de atendimento é fundamental para atender a demanda. Uma possibilidade de reorganização é o atendimento grupal, que tem se mostrado efetivo, ao invés de atendimentos psicológicos individuais (McCrone \& cols., 2005).

\section{Considerações Finais}

Este estudo investigou os fatores de risco da família e as medidas adotadas pela rede após a revelação do abuso sexual. Verificou-se que as pessoas a quem as crianças revelaram o abuso sexual foram os pais e outros familiares na maioria dos casos. Dentre as medidas adotadas pela família para proteção da criança foram identificadas a notificação e o afastamento do agressor. Contudo, em 32,5\% dos casos não foi realizada uma notificação formal aos órgãos de proteção e em $10 \%$ dos casos nenhuma medida de proteção foi adotada pela família. Dentre os casos denunciados, verificou-se que 35\% das meninas foram abrigadas e $20 \%$ dos agressores foram criminalmente punidos. Estes dados apontam que a rede de apoio social e afetiva ainda apresenta significativas dificul- 
dades para desempenhar medidas protetivas que garantam a saúde e o bem estar das crianças e adolescentes.

A falta de capacitação da rede para identificar os casos de abuso sexual e de conhecimento das leis de proteção à criança e ao adolescente são fatores que contribuem para a potencializar os riscos do abuso sexual para a vítima e sua família. Outro fator que contribui para a ineficácia do sistema de proteção é a falta de comunicação e planejamento coordenado de diferentes serviços que compõem a rede. Em alguns casos, a violência é identificada, denunciada, mas a criança não recebe os atendimentos determinados como fatores de proteção, como o acompanhamento psicológico, por exemplo. A rede, incluindo família, escola, conselho tutelar, promotoria e juizado da infância e juventude, abrigos, serviços de acompanhamento social e de saúde, será efetiva quando garantir a segurança e apoio emocional à vítima e ao cuidador não-abusivo responsável pela criança e adolescente. Dessa forma, os serviços e seus profissionais necessitam capacitação contínua e avaliação dos programas de capacitação utilizados para identificação de possíveis falhas, bem como estratégias bem sucedidas de intervenção.

\section{Referências}

Amazarray, M. R., \& Koller, S. H. (1998). Alguns aspectos observados no desenvolvimento de crianças vítimas de abuso sexual. Psicologia Reflexão e Crítica, 11(3), 546-555.

Braun, S. (2002). A violência sexual infantil na família: Do silêncio à revelação do segredo. Porto Alegre: Age.

Briere, J., \& Elliott, D. M. (2003). Prevalence and psychological sequelae of self-reported childhood physical and sexual abuse in a general population sample of men and women. Child Abuse \& Neglect, 27, 1205-1222.

Berliner, L., \& Conte J. R. (1995). The effects of disclosure and intervention on sexually abused children. Child Abuse \& Neglect, 19, 371-384.

Brito, R. C., \& Koller, S. H. (1999). Redes de apoio social e afetivo e desenvolvimento. In A. M. Carvalho (Ed.), O mundo social da criança: Natureza e cultura em ação (pp. 115-130). São Paulo: Casa do Psicólogo.

Cohen, J. A, Mannarino, A. P., \& Rogal, S. (2001). Treatment practices for childhood posttraumatic stress disorder. Child Abuse \& Neglect, 25, 123-135.

Costa, M. C. O., Carvalho, R. C., Bárbara, J. F. R. S., Santos, C. A. S. T., Gomes, W. A., \& Sousa, H. L. (2007). O perfil da violência contra crianças e adolescentes, segundo registros de Conselhos Tutelares: Vítimas, agressores e manifestações de violência. Ciência \& Saúde Coletiva, 12(5), 1129-1141.

Duarte, J. C., \& Arboleda, M. R. C. (2004). Sintomatologia, avaliação e tratamento do abuso sexual infantil. In V. Caballo (Ed.), Manual de Psicologia Clínica Infantil e do Adolescente: Transtornos Gerais (pp.293-321). São Paulo: Santos.

Elliott, A. N., \& Carnes, C. N. (2001). Reactions of nonoffending parents to the sexual abuse of their child: A review of the literature. Child Maltreatment, 6(4), 314-331.

Estatuto da Criança e do Adolescente (1990). Diário Oficial da União. Lei Federal n ${ }^{\circ}$ 8.069, de 13 de julho de 1990, Brasília, DF.
Ferreira, A. L., \& Schramm. F. R. (2000). Implicações éticas da violência doméstica contra criança para profissionais de saúde. Revista de Saúde Pública, 34(6), 659-665.

Fromer, L. (2002). O abrigo: Uma interface no atendimento à criança e ao adolescente vítimas de violência intrafamiliar. In D. C. A. Ferrari \& T. C. C. Vecina (Eds.), O fim do silêncio na violência familiar (pp. 311-315). São Paulo: Ágora.

Furniss, T. (1993). Abuso sexual da criança: uma abordagem multidisciplinar. Porto Alegre: Artes Médicas.

Goodman-Brown, T, B., Edelstein, R. S., Goodman, G. S., Jones, D. P. H., \& Gordon, D. S. (2003). Why children tell: A model of children's disclosure of sexual abuse. Child Abuse \& Neglect, 27, 525-540.

Gomes, R., Junqueira, M. F. P, Silva, C. O., \& Junger, W. L. (2002). A abordagem dos maus-tratos contra a criança e o adolescente em uma unidade pública de saúde. Ciência \& Saúde Coletiva, 7(2), 275-283.

Habigzang, L. F., Azevedo, G. A., Koller, S. H., \& Machado, P. X. (2006). Fatores de risco e de proteção na rede de atendimento a crianças e adolescentes vítimas de violência sexual. Psicologia: Reflexão e Crítica, 19(3), 379-386.

Habigzang, L. F., \& Caminha, R. M. (2004). Abuso sexual contra crianças e adolescentes: Conceituação e intervenção clínica. São Paulo: Casa do Psicólogo.

Habigzang, L. F., Koller, S. H., Azevedo, G. A., \& Machado, P. X. (2005). Abuso sexual infantil e dinâmica familiar: Aspectos observados em processos jurídicos. Psicologia: Teoria e Pesquisa, 21(3), 341-348.

Habigzang, L. F., Koller, S. H., Hatzenberger, R., Stroeher, F., Cunha, R. C., \& Ramos, M. (2008). Entrevista clínica com crianças e adolescentes vítimas de abuso sexual. Estudos em Psicologia, 13(3), 285-292.

Koller, S. H., \& De Antoni, C. (2004). Violência intrafamiliar: Uma visão ecológica. In S. H. Koller (Ed.), Ecologia do desenvolvimento humano: Pesquisa e intervenção no Brasil (pp.293-310). São Paulo: Casa do Psicólogo.

Kristensen, C. H. (1996). Abuso sexual em meninos. Dissertação de Mestrado, Universidade Federal do Rio Grande do Sul, Porto Alegre.

Maniglio, R. (2009). The impact of child sexual abuse on health: A systematic review of reviews. Clinical Psychology Review, 29, 647-657.

Maia, J. M. D., \& Williams, L. C. A, (2005). Fatores de risco e fatores de proteção ao desenvolvimento infantil: Uma revisão da área. Temas em Psicologia, 13(2), 91- 103.

McCrone, P., Weeramanthri, T., Knapp, M., Rushton, A., Trowell, J., Miles, G., \& Kolvin, I. (2005). Cost-effectiveness of individual versus group psychotherapy for sexually abused girls. Child and Adolescent Mental Health, 10(1), 26-31.

Ministério da Saúde Brasil. (2002). Notificação de maus-tratos contra crianças e adolescentes pelos profissionais de saúde: Um passo a mais na cidadania em saúde. Brasília: Ministério da Saúde.

Muthi, M. \& Espelage, D. L. (2005). Childhood sexual abuse, social support, and psychological outcomes: A loss framework. Child Abuse Neglect, 29, 1215-1231.

Runyon, M. K. \& Kenny, M. C. (2002). Relationship of atribucional style, depression and post trauma distress among children who suffered physical or sexual abuse. Child Maltreatment, 7(3), 254-264. 
Sanderson, C. (2005). Abuso sexual em crianças: fortalecendo pais e professores para proteger crianças de abusos sexuais. São Paulo: M. Books do Brasil.

Saywitz, K. J., Mannarino, A. P., Berliner, L,. \& Cohen, J. A. (2000). Treatment for sexually abused children and adolescents. American Psychologist, 55(9), 1040-1049.

Sharma, B. R., \& Gupta, M. (2004). Child abuse in Chandigarh, India, and its implications. Journal of Clinical Forensic Medicine, 11(5), 248-256.
Zavaschi, M. L. S., Telelbom, M., Gazal, C. H., \& Shansis, F. M. (1991). Abuso sexual na infância: um desafio terapêutico. Revista de Psiquiatria/RS, 13(3), 136-145.

Recebido em 26.05.2009

Primeira decisão editorial em 19.07.2010

Versão final em 25.05.2011

Aceito em 14.09.2011

\section{CONGRESSO BRASILEIRO DE PSICOLOGIA DO DESENVOLVIMENTO HUMANO \\ "A PSICOLOGIA DO DESENVOLVIMENTO PARA A TRANSFORMAÇÃO DA AMÉRICA LATINA"}

15 de Novembro de 2011

Brasília, DF, BR 